\title{
Philosophiques
}

\section{Habermas, lecteur de J. L. Austin}

\section{L'illocution et la perlocution dans le modèle communicationnel}

\section{Sébastien Roman}

Volume 43, numéro 2, automne 2016

URI : https://id.erudit.org/iderudit/1038214ar

DOI : https://doi.org/10.7202/1038214ar

Aller au sommaire du numéro

Éditeur(s)

Société de philosophie du Québec

ISSN

0316-2923 (imprimé)

1492-1391 (numérique)

Découvrir la revue

Citer cet article

Roman, S. (2016). Habermas, lecteur de J. L. Austin : l'illocution et la perlocution dans le modèle communicationnel. Philosophiques, 43(2), 441-464. https://doi.org/10.7202/1038214ar

\section{Résumé de l'article}

Dans la Théorie de l'agir communicationnel, Jürgen Habermas élabore pour la première fois le modèle communicationnel, dans l'intention d'en faire la norme de toutes les pratiques langagières. Pour ce faire, il recourt aux analyses austiniennes sur l'illocution et la perlocution, dont il propose une réinterprétation qui prétend parvenir à leur donner un sens adéquat, et les distinguer clairement. Le présent article fait l'examen critique de cette prétention, et démontre que la pragmatique formelle habermassienne n'est pas convaincante.
Ce document est protégé par la loi sur le droit d'auteur. L'utilisation des services d'Érudit (y compris la reproduction) est assujettie à sa politique d'utilisation que vous pouvez consulter en ligne.

https://apropos.erudit.org/fr/usagers/politique-dutilisation/ 


\title{
Habermas, lecteur de J. L. Austin
}

\author{
L'illocution et la perlocution dans le modèle communicationnel \\ SÉBASTIEN ROMAN \\ ENS Lyon \\ sbrom@free.fr
}

\begin{abstract}
RÉSUMÉ. - Dans la Théorie de l'agir communicationnel, Jürgen Habermas élabore pour la première fois le modèle communicationnel, dans l'intention d'en faire la norme de toutes les pratiques langagières. Pour ce faire, il recourt aux analyses austiniennes sur l'illocution et la perlocution, dont il propose une réinterprétation qui prétend parvenir à leur donner un sens adéquat, et les distinguer clairement. Le présent article fait l'examen critique de cette prétention, et démontre que la pragmatique formelle habermassienne n'est pas convaincante.
\end{abstract}

\begin{abstract}
In the Theory of Communicative Action, Jürgen Habermas, for the first time, develops the communicational model, with the intent to take it as the norm of language practices. To this end, he refers to John L. Austin's analysis of illocutionary and perlocutionary acts, and proposes to reinterpret these notions in order to adequately define and clearly distinguish them. The present paper evaluates this Habermasian project, and shows that Habermas' Formal Pragmatics is not convincing.
\end{abstract}

L'éthique habermassienne de la discussion est aujourd'hui très bien connue, et son consensualisme, ainsi que son formalisme, ont et continuent de donner lieu à de nombreux commentaires. Notre intention ici est d'attirer l'attention sur un moment crucial de sa gestation dans la Théorie de l'agir communicationnel ${ }^{1}$, quand Habermas propose une justification de la normativité de l'agir communicationnel par une reprise des travaux de John Langshaw Austin sur l'illocution et la perlocution.

Habermas se sert d'Austin pour faire de l'agir communicationnel le modèle à partir duquel devront être évaluées toutes les pratiques langagières. Pour ce faire, il prétend avoir trouvé le sens véritable de l'illocution et de la perlocution, qui manquait à Austin pour parvenir à les distinguer clairement. Dans Quand dire, c'est faire ${ }^{2}$, Austin n'était pas lui-même satisfait de sa classification des actes de discours, et il est indéniable qu'il n'est pas toujours facile de le suivre dans ses tentatives de distinction des actes illocutoires et perlocutoires. Habermas prétend avoir trouvé le moyen de mettre fin aux hésitations austiniennes par l'élaboration d'un formalisme

1. Jürgen Habermas, Théorie de l'agir communicationnel, Paris, Fayard, I987, tome I. Dans la Théorie de l'agir communicationnel, le modèle de l'éthique de la discussion n'est pas encore (explicitement) élaboré ou construit. Mais les réflexions menées par Habermas sur la normativité de l'agir communicationnel y contribuent fortement, si bien que les contester revient à remettre en cause un tel modèle.

2. John L. Austin, Quand dire, c'est faire, Paris, Seuil, I970. 
qui permettrait de dépasser les embarras liés à une approche empirique du langage qui ne peut que se perdre, selon lui, dans la diversité des actes de parole étudiés.

Par conséquent, l'enjeu est de savoir si Habermas tient parole, autrement dit si son interprétation de l'illocution et de la perlocution est convaincante, non seulement pour reprendre, voire achever les travaux d'Austin, mais également pour ériger en norme l'agir communicationnel. Habermas découvre-t-il le sens véritable des actes illocutoire et perlocutoire, ou n'en propose-t-il qu'une modification substantielle qui, loin de convaincre, révèle le caractère infondé et contradictoire du modèle communicationnel ? La prétention à l'universalité de l'agir communicationnel est-elle légitime, ou n'estelle que l'invention d'un métadiscours qui à la fois occulte l'hétérogénéité des pratiques langagières, introduit une dimension intentionnelle problématique dans l'analyse du langage, et fait abstraction du monde social, notamment de la hiérarchie et des rapports de force qui existent entre les individus ?

Notre raisonnement va procéder en deux temps: nous allons, premièrement, expliciter les raisons pour lesquelles Habermas lit Austin, et la manière dont il le fait. Il sera question, ensuite, d'évaluer la pertinence de son interprétation des actes illocutoire et perlocutoire, en reprenant certains points des discussions très intéressantes que Habermas a eues avec Erling Skjei à ce sujet, et avec Herbert Schnädelbach sur la question de la rationalité communicationnelle, pour les prolonger et les compléter ${ }^{3}$.

\section{L'illocution et la perlocution: leur clarification par la distinction entre l'agir communicationnel et l'agir stratégique}

Rappelons très brièvement, pour commencer, certains points essentiels. La Théorie de l'agir communicationnel est l'ouvrage dans lequel, pour la première fois, Habermas élabore conceptuellement sa théorie de l'activité communicationnelle ${ }^{4}$. Nombre de commentateurs considèrent, avec raison, que cet ouvrage représente un tournant dans la pensée habermassienne, et ne s'opposent ensuite que sur la manière de l'interpréter, soit positivement comme le signe d'un perfectionnement, soit de façon beaucoup plus critique

3. Erling Skjei, «A Comment on Performative, Subject, and Proposition in Habermas's Theory of Communication", Inquiry, $\mathrm{n}^{\circ}$ 28, I985, p. 87-105; Jürgen Habermas, "Reply to Skjei ", Inquiry, n²8, I985, p. I05-II3; "Some Further Clarifications of the Concept of Communicative Rationality ( (1996), in Jürgen Habermas, On the Pragmatics of Communication, Cambridge (Mass.), Mit Press, I998, p. 307-342. Ces textes ne sont pas encore traduits en français. D'où l'intérêt de les commenter aujourd'hui pour en favoriser une plus grande connaissance.

4. Très schématiquement, Habermas s'intéresse de plus près à la théorie de l'activité communicationnelle dès I968, après Connaissance et intérêt qui marque sa rupture avec le modèle de la psychanalyse pour penser l'élaboration des sciences sociales critiques. Mais il faut attendre la Théorie de l'agir communicationnel pour en avoir une modélisation plus précise et rigoureuse. 
— c'est notre cas - comme celui d'un éloignement vis-à-vis des travaux de Max Horkheimer et de Theodor W. Adorno en raison de l'abstraction du modèle adopté.

Pour autant, Habermas ne change pas de projet $^{5}$. Depuis L'espace public, publié en I96I, son intention est de permettre l'exercice d'une démocratie authentique en reprenant l'idéal ou l'idée d'humanité (morale) de la «sphère publique bourgeoise" ou "opinion publique» (Öffentlichkeit) advenue au XviII ${ }^{\mathrm{e}}$ siècle. Une démocratie véritable suppose idéalement, au nom de la dignité humaine, une participation de tous à la vie publique qui prendrait la forme d'un usage public de la raison permettant aux individus de s'abstraire de leurs inégalités sociales et économiques, pour réfléchir ensemble et de manière désintéressée sur ce que doit être une société juste.

La Théorie de l'agir communicationnel est le moment où Habermas forge conceptuellement le moyen de réaliser son projet, en partant de la révolution majeure opérée selon lui par le linguistic turn, à savoir l'élaboration possible d'une pensée post-métaphysique qui dépasse le cadre solipsiste de la philosophie de la conscience. Le tournant linguistique démontre de manière indubitable et définitive la dimension intersubjective de l'identité personnelle. Ce faisant, l'enjeu est de proposer une nouvelle lecture de la rationalité de la modernité après Max Weber, en développant sa dimension communicationnelle contre sa réduction sur le plan strictement instrumental. Habermas désire élaborer une philosophie post-métaphysique qui se nourrit des sciences sociales. La philosophie moderne a perdu son autosuffisance en ne pouvant plus prétendre s'appliquer au monde dans son ensemble. Ce qui la caractérise est désormais d'être une méta-philosophie, une discipline qui doit faire un retour critique sur elle-même pour prendre conscience de ses limites. N'étant plus totalisante, elle a tout à gagner à travailler avec d'autres disciplines, particulièrement la sociologie qui a su conserver une approche globale des phénomènes sociaux tout en étant soucieuse de leur diversité, ce qui la rend capable d'être sensible aux différentes formes de rationalité de l'action. Pour Habermas, la sociologie recourt implicitement — il s'agit d'une catégorisation ou d'une reconstruction qu'il propose, surtout en ce qui concerne l'agir communicationnel, encore trop peu développé par les sociologues en raison du primat de la rationalité instrumentale - à quatre concepts d'action. Les quatre se distinguent en théorie, mais les énoncés linguistiques, en pratique, relèvent très souvent de plusieurs d'entre eux:

- l'agir téléologique, premièrement, comme son nom l'indique, désigne toute action faite en vue d'une cause finale, qui satisfait

5. Il n'y a pas un «premier» et un «second» Habermas, mais un infléchissement de sa pensée par l'adoption du modèle de l'éthique de la discussion. Sur ce point, voir Alexandre Dupeyrix, Citoyenneté et responsabilité, Paris, Maison des Sciences de l'Homme, 20I2, p. I4-I7. 
l'intention de l'agent. Il s'agit nécessairement d'actions orientées vers un "succès égocentrique ${ }^{6}$ ", nommées «instrumentales" ou «stratégiques» selon que l'agent cherche ou non à susciter un effet chez la ou les personne(s) à laquelle (auxquelles) il s'adresse. Seule l'action stratégique désigne la recherche d'une telle influence. Le modèle téléologique d'action devient stratégique, «lorsque l'acteur fait intervenir dans son calcul de conséquences l'attente de décision d'au moins un acteur supplémentaire qui agit en vue d'un objectif à atteindre $^{7}$ ». L'agir téléologique présuppose l'existence d'un monde objectif - «l'ensemble des états de choses qui existent ou se produisent ${ }^{8} »$ - pour que l'individu exprime ses opinions, ou agit comme il désire le faire. Une partie de nos opinions relève de l'agir téléologique. En affirmant notre point de vue, on peut viser un certain succès égocentrique en exprimant notre perception du monde (cas d'une action instrumentale), de même que l'on peut chercher à imposer son avis ou à persuader son auditoire (agir stratégique). Se lever en direction du réfrigérateur pour y prendre quelque chose, par exemple, est une action téléologique. Elle devient stratégique si on manifeste aux autres notre difficulté à nous lever de table, pour qu'ils se lèvent à notre place, après avoir pris en considération leur réaction prévisible à notre égard.

- vient ensuite l'agir normatif ou régulé par des normes, qui concerne «les membres d'un groupe social qui orientent leur action selon des valeurs communes ${ }^{9}$ » faisant l'objet d'un consensus ou d'un accord. De telles actions — d'ordre moral ou juridique, par exemple — supposent en supplément du monde objectif la référence à un monde social (propre à une société donnée). Il s'agit du respect ou de la conformité avec des conventions sociales adoptées, comme par exemple se laver les mains avant de passer à table, finir un message électronique par la formule "très cordialement " pour marquer un respect, etc.

- le troisième cas est celui de l'agir dramaturgique, qui désigne toutes les actions par lesquelles l'agent se met lui-même en scène ou donne à autrui un aperçu de sa subjectivité. Il s'agit d' "autoprésentations » ou de "présentations de soi expressives " (expression de sentiments, d'états psychiques, etc.) qui, pour être comprises dans leur singularité, supposent de différencier le monde subjectif exprimé des deux autres mondes précédemment cités. Par exemple: «je n'aime pas ce vin», «je suis fatigué», ou toute autre assertion par laquelle on

6. Habermas, Théorie de l'agir communicationnel, p. 295.

7. Ibid., p. 101.

8. Ibid., p. I03.

9. Ibid., p. гог. 
présente quelque chose de soi devant un auditoire qui nous sert de public.

- l'agir communicationnel, enfin, concerne toute interaction pleinement intersubjective, en reposant sur l'intercompréhension. Le langage, dans ce cas, n'est ni un moyen de persuasion pour imposer des idées (agir stratégique), ni un médium par lequel sont affirmées et renforcées des valeurs de manière automatique et sans réflexion (agir normatif), ni l'instrument d'une mise en scène de soi. L'agir communicationnel serait le seul à respecter et à révéler la dimension intersubjective du langage. Les locuteurs ne se conduisent plus de manière irréfléchie sans s'interroger sur la pertinence de leurs propos, mais jugent ensemble la manière avec laquelle l'énoncé formulé se rapporte au monde (selon les trois modes cités) et les conditions de sa validité. Chacun d'entre eux est contraint de justifier ses assertions pour qu'elles soient acceptées par les autres. L'agir communicationnel inclut les trois prétentions à la validité que l'on retrouve séparées dans les autres concepts d'action: à savoir la "vérité propositionnelle» (examen de la vérité ou de la fausseté de la proposition ou opinion émise, l'action instrumentale étant aussi soumise au critère de l'efficacité pour ce qui est du succès ou de l'échec de l'action visée); la "justesse normative » (la conformité ou non des actions entreprises aux normes existantes; cas de l'agir normatif); enfin la «véracité » ou "l'authenticité » (selon la sincérité de l'agent; cas de l'agir dramaturgique).

Seul un accord ou un consensus rationnellement motivé permet de savoir si ce qui est dit est justifié. L'argumentation ou discussion (Diskurs) désigne le type de discours dans lequel les interlocuteurs peuvent s'adresser des critiques réciproques pour examiner les prétentions à la validité des arguments énoncés. Elle est régie par quatre principes ou présuppositions idéalisantes (quasi-transcendantales) que tout interlocuteur, s'il décide d'entrer en discussion, est contraint de respecter ${ }^{10}$ : une discussion véritable suppose la recherche commune et désintéressée du meilleur argument. Elle débouche nécessairement sur l'obtention d'un consensus rationnel qui, idéalement, a une dimension universelle (ce qui convainc les partenaires de la discussion doit pouvoir convaincre toute personne). Les trois autres conditions sont la sincérité des partenaires, la symétrie des participants (chacun doit considérer autrui comme son égal, le principe d'égalité d'accès à la parole doit être appliqué et respecté), et leur liberté d'adhésion (choix personnel d'entrer en discussion).

10. Les présuppositions ne sauraient être purement transcendantales, sous peine de rester encore prisonnier d'une philosophie de la conscience. Sur ce point, Habermas s'oppose à Karl-Otto Apel. Nous n'entrerons pas ici dans les détails. 
Habermas juge qu'il ne servirait à rien de recenser les différentes actions langagières, même de manière exhaustive, s'il était impossible de les comprendre toutes à partir d'un même modèle. Une pragmatique formelle est nécessaire pour que nous puissions nous y retrouver dans nos pratiques et ne pas être victimes d'illusions, en sachant distinguer le dialogue authentique - cas de la discussion - des autres modes de discours tronqués. En reprenant ce qu'il appelle la version "officieuse" de la théorie wébérienne de l'action ${ }^{11}$, il réduit à deux les quatre concepts d'action précédemment décrits, à savoir l'opposition entre l'agir téléologique et l'agir communicationnel. C'est là qu'il recourt à la distinction austinienne entre l'illocutoire et le perlocutoire, d'une part pour clarifier la distinction entre ces deux formes d'agir, d'autre part pour démontrer que l'intercompréhension est inhérente au langage (il l'a seulement affirmé jusqu'ici), qu'elle en est son telos (même si c'est loin d'être toujours le cas en pratique), et surtout le "mode original ${ }^{12}$ » à partir duquel on peut comprendre les autres pratiques langagières.

L'erreur d'Austin, toutefois, serait de ne pas avoir suffisamment insisté sur la dimension intersubjective de l'illocution pour la distinguer clairement de l'acte perlocutoire et opposer l'agir stratégique à l'agir communicationnel. Il est juste d'affirmer que toute énonciation est à la fois locutoire et illocutoire. Dire quelque chose est toujours effectuer un acte (sauf exception) $)^{13}$. Mais il ne suffit pas d'insister sur l'illocutoire pour uniquement critiquer l'erreur commune des philosophes de croire que le langage se résume à des affirmations vraies ou fausses. Austin souligne pourtant, dans la $9^{\mathrm{e}}$ conférence de Quand dire, c'est faire, lorsqu'il remet en cause la possibilité que la production de conséquences puisse être un critère de différenciation entre l'illocution et la perlocution, que l'acte illocutoire doit lui-même produire des effets pour réussir, parmi lesquels on trouve la nécessité que la signification et la valeur de l'énonciation soient comprises ${ }^{14}$. "L'exécution d'un acte illocutoire inclut donc l'assurance d'avoir été bien compris [the securing of uptake] ${ }^{15}$." Le caractère conventionnel de l'illocution, qui la distingue de manière plus satisfaisante de l'acte perlocutoire, fait également référence à l'intersubjectivité, car il faut bien que l'on sache de quelle convention il s'agit - que l'on

11. Outre sa version officielle (l'action et ses quatre idéaux-types), s'ajouterait une distinction selon le mécanisme de coordination de l'action, selon que la relation sociale repose sur des situations d'intérêts ou sur un consentement normatif. Toutefois, Weber ne pousse pas jusqu'au bout cette idée, en restant prisonnier de l'approche instrumentale de la rationalité.

12. Ibid., p. 298.

13. Un acte illocutoire est un "acte effectué en disant quelque chose, par opposition à l'acte [locutoire] de dire quelque chose" (Austin, Quand dire, c'est faire, p. I I 3 ). L'acte perlocutoire, lui, revient à faire quelque chose par le fait de dire quelque chose.

14. En plus de devoir être bien compris, l'acte illocutoire doit également "prendre effet, et inviter à "répondre » pour réussir » (Austin, Quand dire, c'est faire, p. I29).

15. Austin, Quand dire, c'est faire, p. I24. 
comprenne les codes qui régissent l'acte, par exemple celui du «Oui, je le veux " prononcé lors de la cérémonie d'un mariage - pour saisir la valeur illocutoire de l'énoncé.

Cependant, Austin n'insiste pas suffisamment sur le critère de l'intercompréhension pour comprendre qu'une discussion réussie vise nécessairement le consensus. Autrement, il aurait précisé que la spécificité de l'acte perlocutoire est volontairement de susciter des effets chez l'auditeur pour satisfaire ses intérêts personnels, dans une intention cachée. L'acte perlocutoire est une négation de la dimension intersubjective du langage pour imposer son propre point de vue. Les effets perlocutoires ne sont possibles que si la dimension illocutoire de l'acte est comprise. Il faut préalablement que l'auditeur comprenne ce que dit le locuteur, et de quelle manière il doit l'interpréter (promesse, ordre, etc.), pour que ce dernier réussisse à le faire réagir comme il le souhaite:

Cependant, les actions langagières ne peuvent servir à cet objectif non illocutoire d'influence sur un auditeur que si elles sont appropriées à l'atteinte d'objectifs illocutoires. Si l'auditeur ne pouvait comprendre ce que dit le locuteur, un locuteur agissant de façon téléologique ne pourrait pas, en recourant à des actes communicationnels, amener l'auditeur à se comporter de la manière souhaitée. Dans cette mesure, ce que nous avions tout d'abord désigné comme «usage du langage orienté vers les conséquences» n'est absolument pas un usage originaire, mais au contraire, la subsomption, sous les conditions de l'agir orienté vers le succès, des actions langagières qui servent d'objectifs illocutoires $^{16}$.

En conséquence, il ne suffit pas de dire, comme Austin, que tous les énoncés ont une valeur illocutoire, pour ne proposer qu'une pragmatique empirique non exhaustive affirmant l'existence de plusieurs «familles" d'actes de discours, «liés entre eux et se recouvrant les uns les autres ${ }^{17} »$. Que l'illocutoire conditionne le perlocutoire prouve que l'agir communicationnel est le modèle à partir duquel les autres registres du discours doivent être compris. L'agir stratégique est une déviation ou une perversion de l'agir communicationnel à des fins de succès personnels. Le consensus est la norme, le critère d'une discussion réussie, et le conflit une pathologie. Bien qu'elle ne soit pas toujours la visée du langage, l'intercompréhension est le modèle à partir duquel on peut comprendre et juger les autres pratiques langagières.

16. Habermas, Théorie de l'agir communicationnel, p. 302.

17. Austin, Quand dire, c'est faire, p. I 53. Il existe, pour Austin, cinq classes de discours, imbriquées les unes dans les autres ou enchevêtrées: «le verdictif conduit à porter un jugement, l'exercitif à affirmer une influence ou un pouvoir, le promissif à assumer une obligation ou à déclarer une intention, le comportatif à adopter une attitude, l'expositif à manifester plus clairement ses raisons, ses arguments, bref à élucider la communication» (Ibid., p. I63). La liste des valeurs illocutoires est nécessairement incomplète car il existe toujours des cas marginaux. Nous n'entrons pas ici dans les détails. 
L'agir communicationnel peut bien avoir des effets, mais ils ne sont pas intentionnels. Ou bien, s'ils le sont - Habermas prend le cas particulier d' "expressions impératives de la volonté", des "impératifs vrais ou des mises en demeure non normativées ${ }^{18}$ » formulés par le locuteur quand il cherche clairement à prendre une position de pouvoir — et si, dans ces situations, l'auditeur n'est pas en mesure d'examiner de manière critique l'ordre donné, alors la dimension intersubjective du langage est niée, et de tels énoncés ne font pas partie de l'agir communicationnel. La spécificité de l'agir communicationnel est de désigner des énoncés dont la prétention à la validité est critiquable. Quand une action est prioritairement orientée vers l'intercompréhension, alors il y a - à différents degrés, selon la prise en considération de la dimension intersubjective de l'acte langagier - une poursuite sans restriction d'objectifs illocutionnaires "afin d'obtenir un accord offrant le fondement d'une coordination consensuelle des plans d'action poursuivis individuellement ${ }^{19}$ ", lequel suppose un examen critique intersubjectif de la valeur de l'énoncé. "Ce qui est important, c'est seulement que la prétention illocutionnaire qu'élève le locuteur pour la validité d'une phrase puisse par principe être critiquée ${ }^{20}$.»

Le couple conceptuel illocutoire/perlocutoire permet de proposer une nouvelle classification des actes de langage qui établit une frontière stricte entre l'agir stratégique et l'agir communicationnel. L'agir normatif et l'agir dramaturgique relèvent de l'agir communicationnel, car ils sont orientés à des degrés divers - vers l'intercompréhension. Ils représentent avec la "conversation ${ }^{21}$ " les trois types purs de l'activité communicationnelle à partir desquels toutes les pratiques langagières doivent être comprises. Toute action que fait un individu sans viser son succès égocentrique relève de l'agir communicationnel. Il dépend de lui d'user des actions régulatrices (agir normatif), expressives (agir dramaturgique), et constatives (conversation), sur un mode prioritairement communicationnel ou stratégique.

\section{Agir communicationnel, agir stratégique: une distinction non convaincante}

Habermas prétend achever le travail d'Austin, en proposant une distinction claire et nette entre l'illocutoire et le perlocutoire, qui permettrait également de les hiérarchiser. De cette manière on pourrait dépasser le caractère pragmatique des cinq classes d'actes de discours - dont l'énumération n'est

18. Habermas, Théorie de l'agir communicationnel, p. 3 I 4.

19. Ibid., 305

20. Ibid., p. 326.

21. La conversation relève des actions langagières constatives. Elle est un contexte d'action, comme l'entretien, l'argumentation, dans lequel il n'est pas question d'activités finalisées. Il est donc maladroit de la réduire à l'agir téléologique. "Je parle toujours de "conversation", lorsque le centre de gravité se déplace ainsi de l'activité finalisée vers la communication. Du fait qu'ici prévaut l'intérêt pour les objets mis en délibération, on peut dire peut-être que les actions langagières constatives ont une signification constitutive pour les conversations» (Ibid.p. 335). 
même pas exhaustive - par l'élaboration d'une pragmatique formelle et normative qui seule permettrait de donner à la communication langagière des fondements rationnels, tout en nous aidant à nous repérer dans nos pratiques langagières, pour ne pas être victimes de duperies ou de mécanismes d'illusion comme peuvent l'être les adolescents par manque d'expérience. Une telle démarche, d'une certaine manière, nous rendrait adultes ou contribuerait à notre maturité:

En s'exerçant aux modes fondamentaux de l'usage du langage, l'adolescent acquiert la faculté de délimiter les frontières entre la subjectivité des expériences vécues propres d'une part, l'objectivité de la réalité objectivée, et d'autre part, la normativité de la société et l'intersubjectivité de la communication langagière elle-même. En apprenant à fréquenter hypothétiquement les prétentions correspondantes à la validité, il s'exerce aux distinctions catégoriales entre essence et manifestation, être et apparence, être et devoir-être, signe et signification ${ }^{22}$.

Une lecture plus approfondie d'Austin montre toutefois que Habermas n'achève pas sa pensée mais la modifie substantiellement. Les modifications apportées donnent davantage la possibilité de critiquer le formalisme de l'agir communicationnel que celle de le justifier. Pour plus de clarté, nous avons distingué plusieurs points dans notre critique:

a) Approche intentionnelle ou structurelle ? Le caractère contradictoire de la normativité de l'agir communicationnel

Dire que l'acte perlocutoire consiste nécessairement à vouloir susciter des effets chez son interlocuteur, de manière insidieuse ou non avouée pour satisfaire ses intérêts personnels, est infidèle au texte austinien. L'effet perlocutoire obtenu peut être intentionnel ou pas, et dans les deux cas il s'agit d'un acte perlocutoire pour Austin. Faire quelque chose par le fait de dire quelque chose ne veut pas dire nécessairement ou exclusivement le faire sciemment et implicitement. On peut convaincre volontairement ou involontairement quelqu'un, ce qui fait que l'acte perlocutoire est plus « « détaché » de l'agent $t^{23}$ » que l'acte illocutoire. On peut aussi «convaincre, persuader, empêcher, et même surprendre ou induire en erreur ${ }^{24}$ » non pas dans son intérêt personnel, mais pour servir une cause. Le perlocutoire désigne les conséquences du discours, en étant tributaire des réactions psychologiques des individus. Les effets produits par les énoncés linguistiques peuvent être et sont la plupart du temps non intentionnels, échappant à notre maîtrise ou

22. Ibid., p. 339-340.

23. Austin, Quand dire, c'est faire, p. 176.

24. Ibid., p. I I9. 
à nos prévisions. Ce que dit Habermas ne vaut que pour une partie de l'acte perlocutoire ${ }^{25}$.

Dans un article de 1985 , Skjei souligne de plusieurs manières le caractère contradictoire de la théorie habermassienne de l'agir communicationnel ${ }^{26}$. Ce qui nous intéresse ici est la contradiction qu'il souligne entre son versant intentionnel et sa prétention d'être une approche purement structurelle du langage, pour en tirer une normativité d'agir. En définissant l'acte perlocutoire comme la recherche volontaire, mais cachée, de produire un effet sur son auditeur pour satisfaire ses propres intérêts, Habermas fait dépendre la distinction entre l'agir communicationnel et l'agir stratégique de l'attitude ou de l'intention du locuteur, contrairement à son projet de démontrer que l'agir communicationnel est, en vertu du langage, le modèle à partir duquel toute pratique langagière doit être comprise. $\mathrm{Si}$, au final, tout dépend de la manière dont je veux utiliser le langage, alors ce n'est pas le langage lui-même qui aurait pour visée l'intercompréhension. La distinction entre l'agir communicationnel et l'agir stratégique ne lui serait pas inhérente, mais dépendrait de mon bon vouloir et de mon éthique. La distinction normative entre les deux agir découlerait de dispositions morales individuelles extrinsèques au langage. Pour Skjei, l'erreur habermassienne est de dire qu'un acte perlocutoire dépend du succès d'un acte illocutoire sans en respecter la normativité, ce qui permettrait de dire que l'agir stratégique est une perversion de l'agir communicationnel.

Or $\mathrm{A}$ peut très bien promettre à $\mathrm{B}$ de faire $\mathrm{x}$, et tenir sa promesse respecter les conditions pour que l'acte illocutoire soit satisfait - tout en le faisant dans une intention cachée, et dans son propre intérêt. Par exemple, promettre à une jolie femme de lui donner un coup de main pour faire du bricolage chez elle, tenir sa parole, et le faire dans l'intention cachée de la séduire, n'enlève rien à la réalisation de notre promesse. Mieux: promettre et ne pas tenir sa parole ne change rien au fait que nous avons promis et que, de par notre acte illocutoire ou performatif, nous nous sommes engagé à la tenir. Habermas ne peut dire le contraire, sans quoi ce serait contraire à ses propres analyses (l'acte illocutoire impose sa propre normativité, il engendre des obligations), de même qu'il s'accorde avec Austin sur les conditions de succès d'un acte illocutoire. L'illocutoire a pour caractéristique nécessaire d'être conventionnel ${ }^{27}$. Il n'y a d'acte illocutoire qu'à condition qu'il soit compris, c'est-à-dire que l'on comprenne à quelle(s) convention(s) l'acte fait

25. Toutefois, après la Théorie de l'agir communicationnel, Habermas modifiera sa conception du perlocutoire, en distinguant trois types d'effets perlocutoires, au lieu de le réduire à l'agir stratégique. Cf. infra p. I 5 .

26. Skjei, "A Comment on Performative, Subject, and Proposition in Habermas's Theory of Communication».

27. Ce n'est pas pour autant sa spécificité. Un acte locutoire peut aussi être conventionnel (exemple: «je vous prie d'agréer mes salutations distinguées» est un énoncé qui respecte une marque de politesse, de respect). 
référence, à quelles(s) condition(s) il la (les) respecte, ce qui revient à connaître ses conditions de félicité: «Il doit exister une procédure, reconnue par convention, dotée par convention d'un certain effet, et comprenant l'énoncé de certains mots de certaines personnes dans certaines circonstances ${ }^{28}$.» La promesse n'a de valeur que si notre interlocuteur comprend que nous lui en faisons une. L'acte illocutoire réussit, si et seulement si notre interlocuteur comprend de notre énoncé son contenu (sa signification), son statut (promesse, hommage, commandement, etc.), et le considère comme valide (il l'est si la convention qui le détermine est respectée; dans le cas de la promesse, il faut pouvoir considérer que le locuteur est sincère, et qu'il désire tenir sa parole $)^{29}$. Tenir pour vrai, juste ou authentique ce qui est dit, selon qu'il s'agit d'un énoncé constatif, normatif, ou expressif, c'est accepter une "offre constituée par l'acte de parole", qui engendre des "obligations d'action du côté du destinataire» (le locuteur, en poursuivant un objectif illocutoire, les a déjà acceptées; en acceptant l'offre, le destinataire s'engage à son tour) «et des expectations d'action correspondantes du côté du locuteur $^{30}{ }$ : l'attitude du destinataire, en acceptant l'offre, devient plus prévisible; le locuteur sait qu'il attend de lui qu'il tienne parole, il lui fait confiance, compte sur lui, et pas sur un autre, etc.

Dans sa réponse à Skjei ${ }^{31}$, Habermas conteste tout versant intentionnel qui rendrait sa théorie contradictoire. L'agir communicationnel découle structurellement du langage, même si son effectivité dépend in fine d'un choix individuel. Que je décide ou non d'entrer en discussion pour rechercher avec mon interlocuteur la vérité sur tel sujet ne change rien à ce que l'intercompréhension soit intrinsèquement la visée de la communication. Et surtout, le succès d'un objectif perlocutoire dépend de la structure de l'acte illocutoire. Il ne peut y avoir de manipulation que parce que la sincérité est attendue, ou que nous laissons croire que nous respectons les règles d'un acte conventionnel, connues de notre interlocuteur, que nous détournons insidieusement. Assurément, dans les deux cas précédemment cités, la promesse est effective. Habermas ne le dit pas aussi clairement, mais cela ne fait aucun doute puisque la Théorie de l'agir communicationnel prouve qu'il s'accorde avec Austin sur les conditions de succès de l'acte illocutoire ${ }^{32}$. Mais cela n'empêche pas la promesse d'être, dans ces deux cas, instrumen-

28. Austin, Quand dire, c'est faire, p. 49.

29. Sur l'importance de la compréhension par notre interlocuteur du caractère conventionnel de l'acte illocutoire, dans la théorie austinienne, cf. Bruno Ambroise, «Illocutoire ou perlocutoire? Retour et détours sur une distinction fondatrice» [https://halshs.archivesouvertes.fr/halshs-OIO9I 859 ], p. 6-7.

30. Habermas, Théorie de l'agir communicationnel, p. 300.

31. Habermas, "Reply to Skjei », p. I07-I08.

32. «Une tentative d'intercompréhension entreprise à l'aide d'un acte de parole réussit lorsqu'un locuteur atteint son objectif illocutoire au sens d'Austin» (Habermas, Théorie de l'agir communicationnel, p. 302). 
talisée dans une perspective stratégique. Austin a raison de dire que l'acte de promettre nous engage tout entier - c'est en promettant que nous promettons - quelle que soit l'intention de tenir, ou pas, sa promesse par la suite. Mais cela ne signifie pas que le critère de l'intention ne compte pas.

Ce sont deux choses différentes que de promettre et d'avoir pour seul objectif que notre interlocuteur comprenne que nous lui promettons quelque chose, et de le faire pour produire un effet perlocutoire, comme moyen en vue d'une autre fin non avouée. L'erreur d'Austin est d' 'identifier les actions langagières, c'est-à-dire, les actes de l'intercompréhension, aux interactions médiatisées par le langage elles-mêmes ${ }^{33}$ ". Il faut distinguer les actions faites uniquement, ou pourrait-on dire prioritairement, dans un objectif illocutoire - prioritairement, car on ne peut empêcher que l'acte illocutoire ait aussi des effets - de celles utilisées comme moyens pour produire des effets perlocutoires. Dans le premier cas, les actions relèvent de l'agir communicationnel, dans le second de l'agir stratégique. Dans un acte perlocutoire, le locuteur masque à son auditeur «le fait qu'il ne remplit pas les présuppositions sous lesquelles seuls les objectifs illocutoires peuvent normalement être atteints $^{34}$ ». La réussite de l'acte illocutoire s'effectue alors au prix d'une tromperie, ce qui fait que l'objectif illocutoire n'est pas atteint normalement, mais par un moyen détourné, par confiscation de la dimension intersubjective du langage, puisque nous cachons à notre interlocuteur l'intention qui motive notre action et la dépasse par un contexte téléologique.

Les précisions apportées par Habermas ne sont pas convaincantes à nos yeux, et l'objection de Skjei demeure pertinente. On peut, pour la prolonger, faire les remarques suivantes. Premièrement, il y a bien chez Habermas une approche prétendument structurelle du langage, qui revient à la thèse que le "langage est en lui-même une méta-institution normative qui oblige ${ }^{35}$ " sans qu'il ne parvienne à la justifier. Le problème est le passage ou le saut toujours non expliqué entre l'affirmation que le langage a une dimension intersubjective, et celle que l'intercompréhension est le mode original et la visée du discours. Que l'illocutoire soit supposé être la condition de possibilité du succès perlocutoire ne permet pas d'en déduire que l'agir stratégique est une perversion de l'agir communicationnel. Ce que Habermas juge constitutif du langage n'est que ce qu'il lui attribue par ses propres considérations morales, selon son attachement et sa conception de l'argumentation, pour en faire la norme ou l'étalon de toute pratique langagière.

33. Habermas, Théorie de l'agir communicationnel, p. 304.

34. Ibid., p. 303 .

35. "His theory of the relation between speech acts (illocutionary acts) and communicative action may be read as a variant of the following thesis: Language is in itself a (normatively) obligating meta-institution» (Skjei, «A Comment on Performative, Subject, and Proposition in Habermas's Theory of Communication », p. 96) 
Certes, dans Droit et démocratie $e^{36}$, Habermas distingue le principe de discussion du principe moral. Les présuppositions idéalisantes de la communication sont censées être neutres moralement, la raison communicationnelle ne visant pas à motiver et à diriger la volonté, mais à examiner uniquement la validité des arguments. Toutefois, en accord avec Skjei, on voit mal comment la distinction entre l'agir communicationnel et l'agir stratégique pourrait échapper à toute considération morale - d'une part, de Habermas lui-même, d'autre part de l'individu, qui a la responsabilité de choisir l'un ou l'autre.

D'où notre deuxième remarque et notre nouveau point d'entente avec Skjei : la théorie habermassienne possède bel et bien un versant intentionnel qui la rend contradictoire. Nous l'avons montré de manière flagrante, de notre côté, quand nous avons comparé Habermas à Austin sur la question de la promesse. La différence entre l'agir communicationnel et l'agir stratégique est uniquement d'ordre intentionnel, et Habermas prétend clarifier et achever la distinction entre l'illocution et la perlocution en lui ajoutant ce critère. Skjei propose certaines corrections de la théorie habermassienne pour lui ôter tout versant intentionnel, en mettant l'accent sur ce qui, pour lui, en constitue le cœur, à savoir qu'un acte de discours est composé de trois éléments (expressif, illocutoire, et propositionnel), liés à trois types de monde (objectif, subjectif, social) et de revendications de validité (vérité propositionnelle, justesse normative, et véracité $)^{37}$. De cette manière, on pourrait reprendre le projet habermassien d'une approche purement structurelle du langage, tout en abandonnant la distinction entre l'agir communicationnel et l'agir stratégique, source de contradiction. La solution ne nous paraît pas pertinente, parce qu'elle laisse de côté l'essentiel de ce que Habermas appellera, après la Théorie de l'agir communicationnel, l'éthique de la discussion. La théorie habermassienne de l'agir communicationnel n'est pas convaincante dans son approche structurelle du langage. Mais, à vrai dire, si son versant institutionnel la rend contradictoire, il a pour mérite d'en révéler le sens véritable. La théorie habermassienne revient à une

36. Jürgen Habermas, Droit et démocratie. Entre faits et normes, Paris, Gallimard, I997.

37. Skjei reproche à Habermas de ne pas arriver à montrer de manière convaincante que le composant illocutoire relève du monde social, l'élément expressif du monde subjectif, et le composant propositionnel du monde objectif. Pour lui, c'est le contenu propositionnel qui détermine a minima le mode de l'énoncé (constatif, expressif, ou normatif). Toutefois, Habermas a raison de lui reprocher de dissocier le composant illocutoire du composant propositionnel. Tous deux font partie de l'acte illocutoire. Un locuteur, en effet, ne peut atteindre son objectif illocutoire que si son auditeur comprend à la fois le contenu de l'énoncé (son élément propositionnel), et son statut (son composant illocutoire). De même Skjei oublie-t-il le plus important, à savoir l'appartenance du «je» au monde vécu, avant sa relation plus secondaire aux trois types de monde. Nous n'entrons pas plus ici dans les détails. 
approche intentionnelle du langage, qui gagnerait à s'assumer comme telle, bien que nous la rejetions.

b) Le caractère extramondain de l'agir communicationnel et la rationalité communicationnelle

Si la théorie de l'agir communicationnel ne nous paraît pas acceptable, c'est aussi parce que, en prenant pour idéalité une certaine conception de la discussion ou de l'argumentation, elle occulte l'ancrage social de l'acte illocutoire, par abstraction des positions sociales des interlocuteurs et des rapports hiérarchiques qui existent entre eux.

Or, pour qu'un acte illocutoire soit bien compris, il faut que les individus prennent en considération le statut social des agents qui le font, pour savoir si c'est avec légitimité qu'ils exercent leur autorité ou leur fonction. Le rituel social auquel fait référence l'acte illocutoire ne sera respecté qu'à cette condition. Par exemple, tout le monde ne peut pas baptiser un bateau, ou légitimement promettre de baisser les impôts, marier un couple, etc. Nous ne sommes pas égaux dans la possibilité de faire des actes illocutoires, de même que tout le monde n'a pas - en raison de son milieu social, son éducation, ses expériences - ni connaissance de tous les actes illocutoires possibles (tout le monde ne s'imagine pas baptiser un bateau), ni connaissance - pour chacun d'entre eux qu'ils savent exister — des règles à appliquer. Austin dit clairement que la force illocutoire des actes de parole n'est pas exclusivement linguistique, mais dépend d'un contexte socioculturel, ou de conditions de félicité dont fait partie la position sociale des locuteurs. Ce point apparaît nettement dans la troisième conférence, quand Austin prend l'exemple de l'ordre "Allez ramasser du bois ", donné sur une île déserte sur laquelle se trouveraient des individus (on peut supposer après un naufrage). Le même énoncé formulé par le capitaine, avant le naufrage, à un subalterne, vaut comme ordre inconditionnel qui ne doit pas être discuté, mais exécuté. Cependant, une fois sur l'île déserte, les rapports d'autorité ne tiennent plus (supposons-le), et il dépend alors du bon vouloir de ce subalterne d'obéir ou non à cet ordre ${ }^{38}$.

La conception habermassienne de l'illocutoire - dont l'expression idéale est l'argumentation ou la discussion - pourrait revenir à ce que Pierre Bourdieu appelle, en reprenant explicitement Austin, la "position scolastique» qui caractérise de manière péjorative la philosophie. Austin

38. Je dois à Bruno Ambroise d'avoir mieux compris l'importance de la position sociale des locuteurs dans l'acte illocutoire, et la possibilité de critiquer sur ce point Habermas, notamment en m'aidant des analyses de Pierre Bourdieu. Cf. Bruno Ambroise, "Une conception non scolastique de l'efficacité linguistique. Bourdieu lecteur d'Austin", in Marie-Anne Lescourret (dir.), Pierre Bourdieu. Un philosophe en sociologie, Paris, Presses Universitaires de France, 2009, p. 65-88; "Le pouvoir symbolique est-il un pouvoir du symbolique? Remarques sur les contradictions du pouvoir symbolique selon P. Bourdieu", Philosophie, $\mathrm{n}^{\circ} \mathrm{II}_{5}, 20 \mathrm{I}_{2} / 4$, p. 75-9I. 
dénonce, par cette expression, à la fois une étude réductrice et simpliste des mots, qui explique que l'on ait ignoré pendant très longtemps le performatif, et une faible considération par les philosophes de la réalité, qui se contentent toujours des mêmes exemples, et certainement des plus simples ou des plus arrangeants, pour justifier leurs théories, au lieu de les tester authentiquement $^{39}$. De façon similaire, Bourdieu dénonce par la position scolastique du philosophe l'absence de réflexion sur les propres présupposés de son attitude et de son discours, qui tiennent à la skholè, le "jeu sérieux» de la pensée auquel il s'adonne, le «temps libre et libéré des urgences du monde ${ }^{40}$ », que le philosophe n'interroge pas de manière critique, mais qu'il justifie au contraire comme étant l'activité noble par excellence, d'où son aristocratisme. La position scolastique du philosophe est son aveuglement sur luimême, l'ignorance de sa propre doxa épistémique (le philosophe a lui aussi ses croyances, ses préjugés), en raison du mépris qu'il a pour la réalité sociale, et surtout pour toute considération des déterminations sociales de son discours. Elle donne au philosophe l'illusion de pouvoir occuper une position neutre, objective, désintéressée, en surplomb du réel tout en pouvant en saisir l'essence, à distance, par la force des concepts ${ }^{41}$.

Habermas appartient à la tradition critique de l'École de Francfort. La théorie critique ne peut être accusée d'ignorer la réalité sociale, puisque son projet est de partir des pathologies sociales que vivent les individus pour les aider à s'émanciper. De même se méfie-t-elle de l'illusion du spectateur impartial, de l'objectivisme qui caractérise la théorie traditionnelle par reconnaissance de son intérêt à l'émancipation. Pour autant, sans travailler le rapport de Bourdieu aux travaux de l'École de Francfort, et le problème de la fidélité de Habermas à l'esprit de la théorie critique, il apparaît ici que le tort de ce dernier est d'éluder une partie considérable de la réalité sociale, en ne tenant pas compte, par exemple, des inégalités de possession de capital symbolique entre les individus. L'accès à la parole n'est pas égal entre les hommes, de même que le pouvoir symbolique, conféré par l'institution à quelques-uns, s'acquiert au prix d'une violence symbolique par exclusion des autres.

Habermas ne nie pas que des rapports de pouvoir existent, ni que le langage soit couramment utilisé à des fins de domination, d'instrumentalisation. Mais il aborde l'illocutoire à partir d'une conception idéalisée d'une intersubjectivité sous la forme de l'accord rationnellement motivé, c'està-dire comme relevant d'énoncés dont la prétention à la validité peut tou-

39. Cf. John L. Austin, Sense and Sensibilia, Oxford, Clarendon Press, I962, p. 3.

40. Pierre Bourdieu, Méditations pascaliennes, Paris, Le Seuil, I997, p. 9.

41. Pour le reproche adressé par Bourdieu à Austin, de critiquer insuffisamment l'illusion scolastique par minimisation des conséquences liées à la skholè, cf. Bourdieu, Méditations pascaliennes, p. 25 ; cf. également Jacques Bouveresse, «Bourdieu, Pascal, la philosophie et la critique de "l'illusion scolastique ", in Fabrice Clément, Marta Roca i Escoda, Franz Schultheis \& Michel Berclaz (dir.), L’inconscient académique, Zürich, Seismo Verlag, 2006. 
jours - sauf exception ${ }^{42}$ et à différents degrés — être critiquée. Les succès illocutoires ne tiennent pas compte des inégalités de position sociale des individus. Ils sont volontairement "extramondains ${ }^{43}$ ", en relevant prioritairement du «monde vécu ", avant la référence aux mondes subjectif, objectif, et social:

Les effets perlocutoires, ainsi que les succès d'actions téléologiques en général, peuvent être décrits comme des états dans le monde, entraînés par une intervention dans le monde. Les succès illocutoires sont en revanche obtenus au niveau de relations interpersonnelles, où des parties prenantes d'une communication s'entendent mutuellement sur quelque chose dans le monde. En ce sens, ils ne sont rien d'intramondains, ils sont - extramondains. Tout au plus, les effets illocutoires adviennent-ils à l'intérieur du monde vécu auquel appartiennent les parties prenantes de la communication, et qui constitue l'arrièrefond de leurs procès d'entente ${ }^{44}$.

Le monde vécu est le concept complémentaire de l'agir communicationnel, qui «assure le rattachement de la théorie de l'action aux concepts fondamentaux de la théorie de la société ${ }^{45}$ ». Il désigne l'ensemble des convictions partagées ou des modèles d'interprétation «transmis par la culture et organisés dans le langage ${ }^{46}$ » qui cimentent la cohésion sociale et servent d'arrièreplan au contexte de l'action. Dans les sociétés traditionnelles, la culture commune était renforcée par la solidarité mécanique. La société était le lieu de l'homogène, et le monde vécu pouvait jouer le rôle d'un consensus d'arrière-plan massif. Ce n'est plus le cas aujourd'hui. Le fait du pluralisme - l'hétérogénéité des valeurs, la confrontation entre diverses orientations axiologiques pour des raisons d'ordre culturel, religieux, etc. - menace de désagréger les liens sociaux. L'espoir ne peut désormais venir que de l'agir communicationnel pour renforcer la solidarité sociale, en permettant aux individus de s'interroger sur ce qui les rassemble et constitue leur identité. Les individus ne partagent plus des évidences mais doivent aujourd'hui justifier leurs arguments pour les rendre valides ${ }^{47}$. La nature intersubjective de l'illocution est rendue possible par la dimension intersubjective du monde vécu, dont l'aspect communicationnel permet de dépasser la hiérarchisation des positions sociales, ou d'en faire abstraction.

42. Cf. infra le cas des impératifs simples, et plus précisément celui de la soumission pure (p. I 5 ).

43. Habermas, Théorie de l'agir communicationnel, p. 303.

44. Ibid., p. 302-303.

45. Ibid., p. 289.

46. Ibid., p. I37.

47. Le concept de monde vécu est originellement husserlien. Mais Edmund Husserl, à tort pour Habermas, n'en a pas compris le renouvellement par la pratique communicationnelle. Nous n'entrons pas ici dans les détails. Sur ce point, cf. Paul Ricoeur, L'idéologie et l'utopie, Paris, Seuil, 1997, p. 288-292; Yves Sintomer, La démocratie impossible? Politique et modernité chez Weber et Habermas, Paris, La Découverte, I999, p. I46. 
Ainsi, Habermas transforme nettement le sens austinien de l'illocution, par une intersubjectivité conçue sur le mode d'une intercompréhension à visée consensualiste. La convention, chez Austin, n'a rien de nécessairement critique, ni ne témoigne nécessairement d'une intersubjectivité «poussée »- dialogale, qui plus est désintéressée - puisque la convention, au contraire, peut être de l'ordre de l'indiscutable, du commandement impératif, tout en relevant de la tradition ou du rituel social respecté(e) par automatisme, ou sans réflexion. Austin ne lui accorde pas différents degrés selon la prise en considération de l'intersubjectivité inhérente au langage, ayant pour norme l'agir communicationnel. Les succès illocutoires, comme les effets perlocutoires, sont intramondains. Ils n'ont pas de dimension intersubjective qui permettrait de dépasser ou de faire abstraction des positions sociales des interlocuteurs ${ }^{48}$.

\section{c) La rationalité de l'agir communicationnel}

La normativité de l'agir communicationnel a toutefois bien été remise en cause par des remarques critiques portant sur la rationalité et les différentes formes qu'elle peut prendre. Le tort de Habermas, selon Schnädelbach, est de concevoir uniquement la rationalité en termes de justification et de critiquabilité. N'est rationnel que ce que l'on peut soutenir pour plusieurs raisons, tout en acceptant que notre interlocuteur puisse en faire un examen critique. Une telle définition de la rationalité la confine à l'argumentation. Or il en existe bien d'autres formes, comme celles de faire l'épreuve du réel selon le modèle freudien, ou bien encore le falsificationnisme dans une perspective poppérienne. Schnädelbach, de son côté, propose de définir la rationalité en termes de capacité d'autoréflexivité personnelle - être en mesure d'expliquer pourquoi nous tenons pour vrai telle assertion, juste telle action, etc.

Dans Some Further Clarifications of the Concept of Communicative Rationality, Habermas réfute d'emblée la définition proposée par Schnädelbach - symptomatique d'une pensée encore métaphysique qui ne tient pas compte de la dimension intersubjective de l'identité personnelle - tout en prenant très au sérieux son objection. Effectivement, concède-t-il, il existe

48. Skjei, lui aussi, critique (indirectement) le caractère extramondain des succès illocutoires. Le «je" pratique, qui relève de l'agir normatif, ne se réfère pas au monde social, mais est dans le monde social, de même que le «je " de l'agir dramaturgique est dans un monde subjectif. Skjei considère que la théorie habermassienne revient à distinguer plusieurs types de «je » selon les trois types de monde; le "je » peut relever soit des énoncés constatifs (le "je » épistémique), soit de l'agir dramaturgique, soit de l'agir normatif. Seul le «je» de l'agir normatif a pleinement une dimension interpersonnelle ou intersubjective, car la relation avec le monde social est plus directe. Comme nous l'avons déjà souligné, Habermas a raison de reprocher à Skjei d'oublier le plus important, à savoir l'appartenance du "je » au monde vécu. En revanche, nous rejoignons Skjei dans son refus d'une position extramondaine du " je », et pour sa conception non nécessaire du lien entre le «je » et l'intersubjectivité. Skjei, "A Comment on Performative, Subject, and Proposition in Habermas's Theory of Communication », p. 96-99. 
plusieurs types de rationalités, et on ne peut se limiter à l'argumentation ${ }^{49}$. Habermas parle de plusieurs "racines », qui sont au nombre de trois: il y a la structure propositionnelle de la connaissance, la structure téléologique de l'action, et la structure communicative du discours. La rationalité discursive n'est pas supérieure aux deux autres en leur servant de fondement, elle joue simplement un rôle d'intégration en établissant entre elles une interrelation $^{50}$. La possibilité d'une interrelation tient à la dimension intersubjective que l'on retrouve, à différents degrés, dans chacune des trois, et qui est rendue possible par la référence au monde vécu. La rationalité épistémique est intrinsèquement ou de nature discursive, des arguments ne peuvent être vrais ou faux que s'ils sont soumis à la critique; de son côté, la rationalité d'un acte n'est pas l'accord entre l'intention et la réalisation, mais la capacité d'expliquer à autrui pourquoi notre action a pu réussir. Enfin, la rationalité communicative prend la forme particulière d'un discours orienté vers l'intercompréhension, dans lequel les prétentions à la légitimité sont soumises à l'examen critique que peuvent en faire les interlocuteurs.

Ainsi, il existe plusieurs types de rationalité dans lesquels l'intersubjectivité est plus ou moins importante ou connaît différents degrés. Dans les perspectives épistémique et téléologique, les expressions linguistiques peuvent et sont souvent employées de manière monologique, mais la dimension discursive reste virtuellement présente. Dans tous les cas, la norme ou la visée de l'accord s'applique. Simplement, l'accord peut connaître différents degrés, selon la reconnaissance de la dimension intersubjective de l'acte illocutoire. Il y a accord, au sens faible ou minimal, quand l'auditeur comprend ce que dit le locuteur, et ses revendications de validité. Avec la rationalité discursive, l'accord est maximal: le locuteur et son interlocuteur s'entendent sur les raisons pour lesquelles ce qui est dit ou fait est valable, après un examen critique intersubjectif des prétentions à la légitimité.

On le voit: si Habermas distingue plusieurs types de rationalité, le raisonnement principal demeure inchangé. Il y a toujours le passage ou le saut de l'intersubjectivité à la normativité de l'agir communicationnel, avec

49. Dans ce qui suit, il n'est pas question de la différence entre la rationalité communicationnelle et la rationalité instrumentale, mais de la conception même de la rationalité que se fait Habermas, et qui ensuite décide de cette distinction.

50. On pourrait croire qu'en refusant un rôle fondateur à la rationalité discursive, Habermas modifie sa théorie, puisqu'en affirmant précédemment que la visée de l'intercompréhension est le «mode original » du langage, l'argumentation semble jouer un tel rôle. De même l'agir communicationnel - et ses trois types purs (conversation, agir normatif, et agir dramaturgique) - sert de modèle aux pratiques langagières et à l'action. En réalité il n’y a pas de changement. Ou plutôt: que tout type de rationalité ne découle pas de celle communicationnelle, ou ne soit pas constitué par elle, génétiquement, n'empêche pas qu'il l'ait pour norme ou idéal, comme nous allons le voir. La capacité de la rationalité discursive d'établir des interrelations entre les différents types de rationalité n'est pas sans rappeler l'existence de "ponts herméneutiques » qu'elle sait établir entre les différents types d'espace public (Habermas, Droit et démocratie. Entre faits et normes, p. 40I). 
le présupposé qu'une discussion réussie débouche nécessairement sur un consensus. Il peut certes exister plusieurs types de rationalité, mais cela ne change rien à la normativité de l'argumentation, et à la position scolastique qui, pour nous, la caractérise. La justification et la critiquabilité restent les critères de la rationalité, Habermas précisant seulement qu'ils existent à des degrés divers dans la connaissance, l'action, et la communication, ce qui lui permet d'étendre le champ du rationnel: si seule l'argumentation au sens strict était rationnelle, alors le champ de l'irrationnel serait immense, comme Schnädelbach avait raison de le reprocher ${ }^{51}$.

Les différences de niveau d'accord ou de consensus aident surtout Habermas à corriger sa conception des impératifs simples ou vrais qu'il avait formulée dans la Théorie de l'agir communicationnel. Skjei a raison de la juger contradictoire car, même dans le cas de tels impératifs qui ne peuvent être évalués en référence directe à des contextes normatifs, un accord minimal est recherché en même temps qu'un succès personnel. L'impératif simple ne peut réussir que si l'auditeur en saisit le sens illocutoire, c'est-àdire comprend l'ordre qui lui est donné, les raisons pour lesquelles son interlocuteur se juge en droit de le formuler, et de l'imposer - au besoin — par des sanctions supplémentaires. En conséquence, Habermas reconnaît son erreur d'avoir classé les impératifs simples (exemple: «Assieds-toi») et les déclarations d'intention («je partirai demain») dans l'agir stratégique. Dans de tels cas, il y a un acte illocutoire sans recherche de consensus, mais dont le mode faible d'intercompréhension assure leur appartenance à l'agir communicationnel. Les raisons d'un impératif simple ne sont peut-être pas "acceptables» - pour cela, il faudrait pouvoir en faire un examen critique

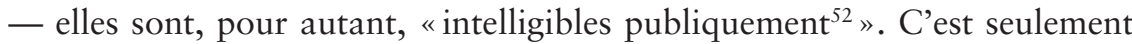
dans le cas extrême d'une pure soumission qu'un impératif relève de l'agir stratégique. Autrement, toute revendication d'autorité ou de pouvoir sup-

51. Une autre manière de critiquer Habermas serait d'insister davantage sur la différence entre la sémantique et la pragmatique. Une étude plus approfondie des différents types d'actes de langage (selon la catégorisation commune entre les assertifs, directifs, commissifs, expressifs, et déclaratifs) permettrait de mieux distinguer ce que chacun d'entre eux, par convention, implique ou suppose, de ce que Habermas leur ajoute avec sa théorie de l'agir communicationnel. Par exemple, affirmer que $\mathrm{P}$ est exprimer la croyance que " $\mathrm{P}$ », supposer que « $\mathrm{P}$ » est justifié, et que « $\mathrm{P}$ » est vrai sur la base de cette justification. Mais cela n'implique pas forcément - comme l'affirme Habermas - que le locuteur en question sache pour quelles raisons précises il pense que " $\mathrm{P}$ » est vrai, ni qu'il ait envie de soumettre son assertion à un examen critique. Habermas impose donc des normes aux assertifs qui leur sont étrangères. Si nous ne faisons pas, dans cet article, une analyse approfondie et centrale des différents types d'actes de langage, en privilégiant le développement d'autres arguments, nous espérons toutefois y parvenir partiellement, par l'étude de cas concrets comme ceux de la promesse et des impératifs simples.

52. "We might call such reasons - to distinguish them from generally acceptable reasons - "publicly intelligible" reasons.» (Habermas, "Some Further Clarifications of the Concept of Communicative Rationality ", p. 322). 
pose une reconnaissance minimale des prétentions à la légitimité, et leur examen possible.

Habermas dit que son erreur a été d'établir une distinction nette entre les impératifs autorisés normativement et les impératifs simples. En réalité, entre une relation de pouvoir habituel et un impératif justifié normativement, il n'y a pas de séparation mais une différence de degré, étant donné que tout impératif est lié — plus ou moins directement, là est la différence - à des contextes normatifs. Il est donc plus juste d'affirmer qu'un acte illocutoire dont l'effet est intentionnel, sans examen critique possible des revendications de validité, appartient à l'agir communicationnel plutôt qu'à l'agir stratégique, comme dit précédemment dans la Théorie de l'agir communicationnel (sauf cas extrême de la soumission pure) (53 $^{53}$ Il en est de même quand il produit un effet de manière non intentionnelle. Après la Théorie de l'agir communicationnel, Habermas modifie sa conception de la perlocution en distinguant trois classes d'effets perlocutoires ${ }^{54}$. Il y a d'abord les effets perlocutoires qui résultent grammaticalement du succès de l'acte illocutoire (par exemple les obligations et les expectations d'action nées de la réussite de l'offre de promesse). Dans ce cas, les objectifs illocutoires déterminent et priment sur les effets perlocutoires, lesquels découlent de ce qui est $\mathrm{dit}^{55}$. Les effets perlocutoires peuvent ensuite être grammaticalement non régulés, en advenant comme des conséquences contingentes d'un acte de langage selon la réaction psychologique imprévisible de l'auditeur. Enfin, les effets perlocutoires relèvent de l'agir stratégique quand ils sont recherchés de manière non déclarée par un individu pour satisfaire ses intérêts.

La reconnaissance de plusieurs effets perlocutoires possibles ne change rien à l'essentiel de la théorie de l'agir communicationnel. L'agir communicationnel et l'agir stratégique divergent toujours en fonction de la priorité accordée entre l'objectif illocutoire et les effets de l'action entreprise. Si mon intention première est de produire un effet sur mon auditeur, par le fait de lui dire telle chose, en lui cachant l'objectif réellement poursuivi, alors je fais un acte perlocutoire qui relève de l'agir stratégique. Là est l'idée principale. D'une certaine manière, Habermas semble faire une distinction entre l'acte

53. Cf. supra p. 6.

54. Habermas, "Some Further Clarifications of the Concept of Communicative Rationality", p. 330.

55. "As long as the intended effect on the hearer follows from what is said, I include it in the "obligations relevant to the sequel of interaction" which the hearer enters into when he accepts the offer, or proposal, of the speech act» (Habermas, Reply to Skjei, p. I06). Bruno Ambroise rappelle également que le terme "convention» - opposé aux réactions supposées «naturelles» ou psychologiques des personnes, quant aux effets sur elles des énoncés formulés — signifie que l'effet illocutoire peut lui-même être conventionnel. La promesse n'a rien de naturel, non au sens qu'il ne serait pas naturel à l'homme de promettre, mais parce que l'acte de promettre obéit à des conventions, à des rituels propres à chaque société. C'est flagrant dans le cas du baptême d'un bateau, par lequel on lui donne un nom. Cf. Ambroise, "Illocutoire ou perlocutoire? Retour et détours sur une distinction fondatrice», p. 6. 
perlocutoire et l'effet perlocutoire - même si cela peut paraître étrange ou maladroit de parler ainsi, puisque, dans le cas du perlocutoire, l'acte consiste précisément à produire un effet par le fait de dire quelque chose. Le changement apporté après la Théorie de l'agir communicationnel consiste seulement à qualifier de "perlocutoires" des effets possibles de l'acte illocutoire que Habermas, jusqu'ici, se refusait à nommer ainsi, sans rien changer de son appartenance à l'agir communicationnel. La distinction entre l'acte et l'effet tient à l'objectif poursuivi — prioritairement illocutoire (agir communicationnel) ou perlocutoire (agir stratégique). Quand nous disons «Assiedstoi ", nous voulons évidemment produire un effet (une certaine obéissance chez notre auditeur), mais nous voulons prioritairement que notre objectif illocutoire réussisse, que notre ordre soit compris et accepté comme tel selon différents degrés d'acceptation, de la soumission à l'examen critique poussé. L'obéissance que nous pouvons obtenir peut découler du succès de l'objectif illocutoire poursuivi. Ou bien on peut engendrer le même sentiment, ou un autre, en raison de la réaction psychologique contingente de notre auditeur, lequel est alors obtenu secondairement par rapport à notre objectif.

d) Le succès illocutoire n'est pas la condition de possibilité de l'effet perlocutoire

Enfin, nous avons vu que, pour Habermas, la dimension illocutoire doit être comprise pour que l'effet perlocutoire visé puisse être obtenu, ce qui lui permet de conclure que l'illocutoire est la condition de possibilité du perlocutoire, et que l'agir stratégique est une perversion de l'agir communicationnel, une confiscation de la dimension intersubjective du langage à des fins personnelles. Par exemple, pour qu'un enseignant obtienne de ses élèves qu'ils se taisent, après leur avoir dit "Taisez-vous ", il faut bien qu'ils comprennent l'ordre de se taire, afin de mettre en adéquation leur comportement avec l'impératif formulé.

Est-il exigé, pour autant, que l'acte illocutoire soit nécessairement compris pour qu'il y ait un effet illocutoire, comme l'affirme Habermas? On peut en douter. D'une part, comme le rappelle Bruno Ambroise, si un acte de parole relève à la fois du locutoire, de l'illocutoire, et du perlocutoire (sauf exception), il serait faux de croire que les trois sont interdépendants. L'effet perlocutoire n'est pas nécessairement une conséquente directe de l'acte illocutoire, de la même manière qu'un acte illocutoire est réussi indépendamment des effets perlocutoires qu'il peut engendrer. Bruno Ambroise prend l'exemple de l'énoncé "Je t'aime" dans le sens d'une déclaration (d'amour). Si cet énoncé émeut la personne à qui il est adressé, ce n'est pas directement à cause de l'acte de parole réalisé - la déclaration — mais principalement en raison des sentiments que cette personne éprouve pour l'auteur de l'énoncé, et qu'ils partagent tous deux. De même la déclaration d'amour est réussie au moment même de sa formulation (si les conditions de 
félicité sont réunies), indépendamment de la (des) réaction(s) qu'elle engendre ${ }^{56}$.

D'autre part, l'acte perlocutoire (au sens austinien) peut tout à fait réussir quand bien même notre interlocuteur n'a pas compris l'acte illocutoire effectué. Reprenons l'exemple du professeur qui demande à certains de ses élèves de se taire. Supposons que les élèves concernés (deux par exemple) n'aient pas compris que l'ordre "Taisez-vous" leur était adressé, ou qu'ils aient cru qu'il concernait d'autres de leurs camarades. Supposons toutefois qu'ils se taisent, soit parce qu'ils comprennent qu'il s'agit d'un ordre qui pourrait leur être adressé, soit parce qu'ils ont eux-mêmes décidé d'arrêter de bavarder, et que cela correspond - heureux hasard — avec l'intention de leur enseignant. L'effet escompté ayant été obtenu, on peut tout à fait dire que l'acte perlocutoire, dans ces deux cas, est réussi. Les élèves peuvent très bien parvenir à se taire effectivement, sans que l'acte illocutoire réussisse et soit satisfait ${ }^{57}$. Dans notre exemple, les conditions de performance de l'acte illocutoire ont échoué, puisque l'ordre n'a pas été reconnu comme ordre. Et l'acte directif donné n'est pas non plus satisfait, puisque l'obéissance obtenue des élèves ne tient pas au directif donné mais à d'autres motifs ${ }^{58}$.

Prenons un dernier exemple. Supposons cette fois-ci que l'enseignant dise «Taisez-vous » à ses élèves (à l'ensemble des élèves qui bavardent dans la classe), dans l'intention non avouée d'être entendu par ses collègues qui font cours dans des salles avoisinantes, pour leur montrer qu'il a de l'autorité, qu'il n'est pas dépassé par sa classe (qui peut être difficile), ou bien pour leur faire croire que lui, avec cette classe difficile, il se débrouille mieux qu'eux, qui l'ont aussi en charge. Il s'agit, dans ce cas, d'un impératif simple de l'ordre de l'agir stratégique (ils exigent de la part de ses élèves une pure soumission, et il leur donne cet ordre en vue de parvenir à une fin qu'ils ignorent). Les collègues peuvent très bien éprouver le sentiment recherché sans que l'acte illocutoire ne réussisse et ne soit satisfait: ce peut être parce que des flocons de neige, tout d'un coup, tombent en abondance que les élèves cessent de parler pour entrer dans un moment de contemplation; ou bien encore parce que quelqu'un, au moment même où l'enseignant leur a crié «Taisez-vous» — ordre dont ils n'ont que faire - a frappé à la porte, et qu'ils en ont été très intrigués (craignant, peut-être, que quelqu'un de la

56. Cf. Bruno Ambroise, Les pouvoirs du langage: la contribution de J. L. Austin à une théorie contextualiste des actes de parole, thèse de doctorat en philosophie, soutenue à Paris X-Nanterre en 2005 sous la direction de Francis Wolff [https://tel.archives-ouvertes.fr/tel00338 I 76 , p. I47-I 48 ].

57. Nous reprenons ici la distinction classique entre les conditions de performance réussie d'un acte, et celles de sa satisfaction.

58. Sur l'absence de satisfaction de l'acte illocutoire réalisé, dans de tels cas, cf. Christian Brassac, "Analyse de conversations et théorie des actes de langage ", Cahiers de linguistique française, $\mathrm{n}^{\circ}$ 13, I992, p. 68; Michel Seymour, L'institution du langage, Montréal, Presses de l'Université de Montréal, 2005, p. 327. 
direction se déplace, ayant conscience qu'ils font trop de bruit). L'effet perlocutoire recherché, pour autant, peut tout à fait être obtenu. Les collègues en question peuvent ne pas avoir compris qu'il s'agissait d'un ordre, ils n'ont pas compris les mots prononcés, mais ont été impressionnés par le ton employé par leur collègue; ou bien encore ils peuvent avoir l'impression qu'il se débrouille mieux qu'eux avec cette classe, non en raison du directif formulé (qui, en plus, de ne pas être compris, peut ne pas être suivi d'obéissance; les élèves peuvent continuer à bavarder), mais parce qu'ils n'ont pas encore entendu de portes qui claquent, de chaises voler, de chahut incessant de la part des élèves, alors même qu'il les a à une heure difficile, en fin de journée, et s'efforce de leur transmettre un savoir, de les intéresser (ils l'entendent faire cours, s'échiner à leur faire lire un texte, ou tel auteur, et c'est cela qui les impressionne).

\section{Conclusion}

Habermas n'achève pas les travaux d'Austin, au sens de leur donner l'aboutissement qui leur manquerait, par l'élaboration d'une distinction claire et nette entre l'illocution et la perlocution. Il ne distingue l'acte illocutoire de l'acte perlocutoire qu'au prix d'une modification de leur sens, en plaquant sur eux les registres de l'agir communicationnel et de l'agir stratégique, ainsi que leur hiérarchie. La théorie de l'agir communicationnel a un versant intentionnel qui la rend contradictoire avec sa prétention de découler de la normativité intrinsèque du langage. Elle occulte également de l'illocution son ancrage social, comme elle a pour défaut de restreindre la rationalité au modèle de l'argumentation, et d'affirmer que l'acte perlocutoire suppose nécessairement un succès illocutoire.

Le consensualisme habermassien, d'une manière plus générale, est absolument étranger à Austin. Quand dire, c'est faire ne contient pas l'idée que l'intersubjectivité ou l'intercompréhension vise nécessairement le consensus, ou qu'une discussion réussie débouche nécessairement sur un accord. Ce n'est pas ce qu'Austin aurait dû comprendre, mais bien ce qu'il ne dit pas et ne peut pas dire du fait de son approche empirique des actes de langage, qui, loin de juger le comportement des individus ou leur usage du langage par référence à une norme, insiste au contraire sur le caractère aléatoire et imprévisible de leurs réactions, autrement dit sur leur liberté.

Habermas affirme que la normativité de l'éthique de la discussion n'est que la prise de conscience réflexive de ce que discuter veut dire, qui débouche sur la connaissance de présuppositions idéalisantes que personne ne peut rejeter sans commettre de contradiction performative. L'idéalité de la discussion serait déjà inscrite dans le fait langagier, quand bien même les rapports de domination et de pouvoir, en pratique, en empêchent l'actualisation. Or il apparaît, de manière plus évidente, que l'importance de l'entente tient plus à la conception que Habermas se fait du langage qu'au langage lui-même. Le modèle de l'agir communicationnel ne nous rend pas plus mûrs dans nos 
pratiques langagières, en nous préservant de toute illusion ou de duperie. Au contraire, on court avec lui le risque d'une illusion plus grande, qui consiste à oublier le monde social et notre place dans ce monde. Il est vrai qu'à la lecture de Quand dire, c'est faire, on est un peu perdu dans la complexité ou le labyrinthe de toutes les dimensions des actes de langage. Mais il vaut mieux l'être, quitte à avancer lentement ou à ne pas avancer du tout, que de se frayer un chemin qui, aussi clair soit-il, ne va pas dans la bonne direction. 